\title{
BMJ Open Outcomes in hospitalised patients with sepsis, severe sepsis or septic shock and reported penicillin allergy: a retrospective cohort study
}

\author{
David Beddow, ${ }^{1}$ Love Patel (D) , ${ }^{1}$ Claire S Smith, ${ }^{2}$ Justin Kirven, ${ }^{1}$ \\ Christine Schmidt, ${ }^{3}$ Daniel Ruppman, ${ }^{1}$ Rajesh Kethireddy, ${ }^{1}$ Michael Wankum, ${ }^{4}$ \\ Barite Dawud, ${ }^{2}$ Catherine A St. Hill (D) ${ }^{2}$
}

To cite: Beddow D, Patel L, Smith CS, et al. Outcomes in hospitalised patients with sepsis, severe sepsis or septic shock and reported penicillin allergy: a retrospective cohort study. BMJ Open 2022;12:e050879. doi:10.1136/ bmjopen-2021-050879

- Prepublication history for this paper is available online. To view these files, please visit the journal online (http://dx.doi. org/10.1136/bmjopen-2021 050879).

Received 02 March 2021 Accepted 03 February 2022

Check for updates

(c) Author(s) (or their employer(s)) 2022. Re-use permitted under CC BY-NC. No commercial re-use. See rights and permissions. Published by BMJ.

${ }^{1}$ Internal Medicine, Allina Health, Minneapolis, Minnesota, USA

${ }^{2}$ Care Delivery Research, Allina Health, Minneapolis, Minnesota, USA

${ }^{3}$ Graduate Medical Education, Allina Health, Minneapolis,

Minnesota, USA

${ }^{4}$ Pharmacy, Allina Health, Minneapolis, Minnesota, USA

Correspondence to

Dr Love Patel;

love.patel@allina.com

\section{ABSTRACT}

Objective To determine outcomes in hospitalised patients with sepsis and reported penicillin allergy (PcnA).

Design Observational retrospective cohort study using data from electronic health records.

Setting A large single health system with 11 hospitals of small, medium and large sizes including a 630-bed tertiary care teaching hospital.

Participants Patients $(n=5238) \geq 18$ years of age, hospitalised with sepsis, severe sepsis or septic shock between 1 January 2016 and 31 December 2018, received antibacterial agents, and had documented PcnA status. Patients $<18$ years of age at admission were excluded. Outcome measures Primary outcomes evaluated were inpatient mortality and 30-day mortality posthospital discharge. Secondary outcomes were hospital length of stay, 30-day readmissions, duration of antibiotic use, rate of Clostridium difficile infection and total cost of care.

Results There was no difference in outcomes including inpatient or 30-day mortality, hospital length of stay, inhospital antibiotic duration, $C$. difficile infection, total cost of care and 30-day readmission rate between patients labelled with a PcnA vs patients who did not report PcnA (non-PcnA).

Conclusion In this retrospective single health system study, there was no difference in key outcomes including inpatient or 30-day mortality in patients admitted with sepsis and reported PcnA compared with patients who reported no PcnA.

\section{INTRODUCTION}

Sepsis is a life-threatening organ dysfunction caused by dysregulated host responses to infection, ${ }^{12}$ with high patient morbidity and mortality. ${ }^{3}$ In the USA, in 2013, cost of care was US\$20 000-US\$40 000/hospitalised patient and US\$23.7 billion dollars annually. ${ }^{45}$ Survivors suffer from long-term physical and cognitive disabilities leading to additional health implications. ${ }^{6}$

In bacterial sepsis, early and appropriate antibiotic administration improves outcomes. $^{7-10}$ Reported antimicrobial

\section{Strengths and limitations of this study}

- Large retrospective electronic medical record-based data from single health system is analysed in this study.

- Authors targeted reported penicillin allergy and its effect on health outcomes in sepsis which is an important area of further research.

- The measurement of outcomes may have been less accurate and consistent than would have been attained with a prospective study design.

- Patients who have not reported penicillin allergy may still be allergic to penicillin.

- The cohorts used in the study may not have been representative of an entire population of patients but instead those seen within our healthcare system.

allergies are considered in antimicrobial regimen selection. ${ }^{11}$ Nationally, $\sim 20 \%$ of hospitalised patient's records describe a penicillin allergy $(\mathrm{PcnA})$, but $<10 \%$ with a reported PcnA have a true clinically relevant allergy when objectively evaluated with rigorous skin testing. ${ }^{12} 13$ PcnA may become part of a patient's history or medical record even if the documented reaction was due to other medications or was non-immune mediated. Patients with reported childhood PcnA are reluctant to receive penicillins although they are low risk for true allergy. Those PcnA patients with negative skin testing usually tolerate penicillins well without adverse outcomes. ${ }^{14}$

Overall cross reactivity is low between penicillins, cephalosporins and carbapenems for PcnA patients. ${ }^{15} 16$ Antibiotic specific risks depend on the side chain structure. ${ }^{17}$ Betalactam antibiotics are among the leading causes of drug induced anaphylaxis leading clinicians instead to administer alternative antibiotics that displace first line therapies 
and increase adverse events in PcnA patients. A PcnA label is associated with increased antibiotic resistance due to increased use of broad-spectrum and non-beta lactam antibiotics. $^{18}$

PcnA patients are more likely to receive carbapenems, fluoroquinolones, clindamycin and vancomycin. ${ }^{10}{ }^{18}$ In a large study hospitalised PcnA patients had increased rates of methicillin-resistant Staphylococcus aureus (MRSA) (2.6\% vs $2.2 \%)$ and vancomycin-resistant Enterococci $(0.6 \%$ vs $0.5 \%$ ) than those without PcnA. ${ }^{19}$ PcnA patients can experience a $50 \mathrm{~min}$ delay in antibiotic initiation, ${ }^{20}$ and more surgical site infections likely because of suboptimal coverage from second-line antibiotics. ${ }^{21}$ Other reported adverse outcomes include increased rates of Clostridium difficile infection and longer hospital stays. ${ }^{19}$ Outcomes are relatively unknown for patients admitted with severe infections and reported PcnA. Potential explanations are that hospitalised sepsis patients frequently receive different antibiotic classes. Penicillins alone are uncommonly used even without reported allergy in patients presenting with sepsis.

Recently, robust antimicrobial stewardship programmes (ASPs) have been implemented to address appropriate and timely antibiotic choices. The Centers for Disease Control and Prevention recommend that all hospitals have ASPs to minimise adverse events and reduce antibiotic resistance. ${ }^{22}$ Some ASPs also incorporate PcnA skin testing proven to be safe and effective even for critically ill patients. ${ }^{23}$ Our research question was, 'What are the health outcomes of inpatients with sepsis, severe sepsis, and shock who were labelled as having PcnA compared with inpatients who were not labelled as having PcnA?' Subsequently, the objective of this study was to compare antibiotic use patterns and outcomes between patients with reported PcnA and non-PcnA hospitalised with sepsis, severe sepsis, and septic shock in a health system with active ASPs.

\section{METHODS}

\section{Study population and data collection}

Hospitalised eligible patients $\geq 18$ years were identified from electronic medical records (EMRs) at an 11-hospital system in Minnesota and Western Wisconsin. Patient's data were collected from 1 July 2016 to 30 June 2018 from our organisation's electronic data warehouse. The study's sample size was obtained after the initial data extract was verified to include all eligible participants. Selection bias was minimal because we included all eligible patients in the cohorts with and without a PcnA label. There was no direct involvement of patients or the public in design and conduct of the study, choice of outcome measures, recruitment or dissemination of the results because of the retrospective study design. The research question was developed based on self-reported PcnA status by hospitalised patients at admission. This information was entered into their medical records and informed provider's medical decision making even though published evidence indicates that the majority of these patients do not have true clinically relevant allergy when objectively tested through rigorous skin testing. ${ }^{112} 24$

\section{Study design}

Patients were selected by electronic health record query of diagnosis codes containing sepsis, severe sepsis or septic shock during specific hospital encounter. Patients with labelled PcnA were compared against those who did not have PcnA label (Non-PcnA). Our rationale was that choices of second line antibiotics in reported PcnA can cause adverse outcomes. Those who did not receive antibiotics were excluded because sepsis can be caused by non-bacterial infections and a reported PcnA may not change the choice of antimicrobial agent. In our health system larger referral hospitals frequently get patient transferred from other hospitals and emergency department outside our health system and data on antibiotic administered before transfer (in emergency department) was not available in some patients. Patient with missing complete antibiotics data were excluded (figure 1). Age, gender, race, body mass index, insurance coverage, risk of mortality, severity of illness and LACE score (to evaluate risk of readmission) were the covariates. Primary outcomes evaluated were inpatient mortality and 30-day mortality posthospital discharge. Secondary outcomes were hospital length of stay (LOS), 30-day readmissions, duration of antibiotic use, rate of $C$. difficile infection and total cost of care.

\section{Statistical analysis}

Patients were categorised as reported PcnA and nonPcnA. Subgroup analysis was performed on PcnA patients who received cephalosporin alone, cephalosporin with another class of antibiotics, or antibiotics other than a cephalosporin. In the PcnA subgroup, patients who received penicillins were excluded. Inpatient and 30-day post discharge mortality, hospital LOS, total cost of care, antibiotic duration, C. difficile infection in hospital and 30-day readmission were evaluated. Means, SD, medians and $\mathrm{Q} 1-\mathrm{Q} 3$, frequencies and proportions, were used to describe the PcnA and non-PcnA groups' patient characteristics and outcomes, as appropriate. Comparisons between the PcnA and non-PcnA groups were conducted using two-sample independent t-tests, Mann-Whitney U tests, $\chi^{2}$ tests and Fisher's exact tests. The descriptive analysis of the three PcnA subgroups were conducted likewise to the main analysis. One-way analysis of variance, $\chi^{2}$ and Kruskal-Wallis tests were conducted, as appropriate, to compare patient characteristics and outcomes across the three PcnA subgroups. Pairwise comparisons were conducted using a Bonferroni correction.

Adjusted analysis was conducted with the primary and secondary outcomes for both the main and the subgroup analysis to address potential bias in estimated effects of a PcnA label. To reduce multicollinearity, risk of mortality and severity of illness variables were both recoded into two categories: 'minor and moderate' and 'major and 


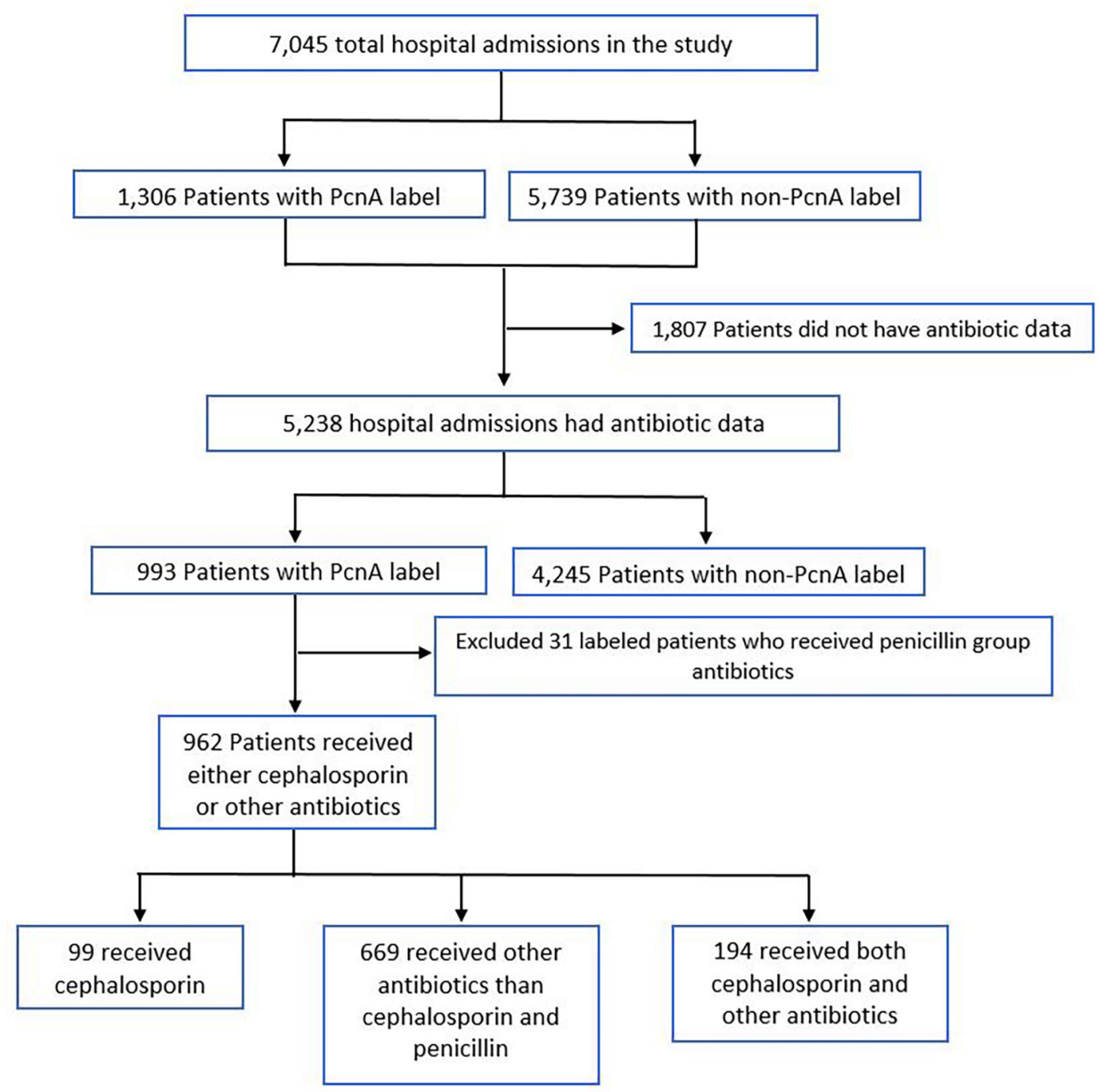

Figure 1 Study population identification. PcnA, penicillin allergy.

extreme'. Logistic regression was used to test for differences in inpatient mortality, $C$. difficile in hospital and 30-day readmission between the PcnA and non-PcnA groups. Unadjusted and adjusted ORs and their respective 95\% CIs were calculated. Potential confounding variables for each model were selected based on clinical importance and a $\mathrm{p}<0.2$ in the unadjusted tests. These variables were further controlled using a backward elimination method. The Firth logistic regression method was used to analyse the inpatient outcomes mortality and $C$. difficile due to the small number of events. Differences between the PcnA and non-PcnA groups in hospital LOS, total cost of care and duration of antibiotics were tested using negative binomial regression and the same model selection process as stated for the logistic regression models.

Regression analysis was also used to test for differences among the three antibiotics subgroups. Inpatient mortality and 30-day readmission were all analysed using Firth logistic regression in unadjusted models. Unadjusted ORs and their respective 95\% CI were calculated. Differences among the subgroups in rates of 30-day readmission were also tested in an adjusted Firth logistic regression model. Differences among the three antibiotics subgroups in hospital LOS, total cost of care and duration of antibiotics were tested using negative binomial regression. Confounders for all adjusted regression models were selected in the same manner as described in the main analysis. Wald tests were used to test for the significance of the three (1) category antibiotics variable as a whole in each model. Two-tailed $p$ values were computed for all tests, with significance at $p<0.05$. All statistical analyses were conducted using $\mathrm{R}$ software V.3.6.1 (The R Foundation for Statistical Computing, Vienna, Austria.)

\section{Patient and public involvement}

There was no direct patient involvement. Data were used from electronic database.

\section{RESULTS}

We identified 7045 hospitalised patients with a documented diagnosis of sepsis, severe sepsis, or septic shock. 


\begin{tabular}{|c|c|c|c|}
\hline & No PcnA label & PcnA label & $P$ value \\
\hline$n$ & 4245 & 993 & \\
\hline BMI, mean (SD) & $30.2(8.9)$ & $31.3(10.5)$ & $0.002^{*}$ \\
\hline White race, $\mathrm{n}(\%)$ & 3709 (88.5) & 931 (94.3) & $<0.0001^{*}$ \\
\hline Male, n (\%) & $2308(54.4)$ & $404(40.7)$ & $<0.0001^{*}$ \\
\hline $\begin{array}{l}\text { Current or former } \\
\text { tobacco use, } n(\%)\end{array}$ & 2361 (59.1) & $591(62.9)$ & $0.0327^{*}$ \\
\hline $\begin{array}{l}\text { Private insurance } \\
\text { status, n (\%) }\end{array}$ & 1120 (26.4) & $265(26.7)$ & 0.846 \\
\hline $\begin{array}{l}\text { Risk of mortality, } n \\
(\%)\end{array}$ & & & 0.632 \\
\hline Minor & $471(11.2)$ & $98(9.9)$ & \\
\hline Moderate & $786(18.6)$ & 195 (19.8) & \\
\hline Major & 1556 (36.9) & $358(36.3)$ & \\
\hline Extreme & 1406 (33.3) & $336(34)$ & \\
\hline $\begin{array}{l}\text { Severity of illness, } n \\
(\%)\end{array}$ & & & 0.231 \\
\hline Minor & $133(3.2)$ & $22(2.2)$ & \\
\hline Moderate & $960(22.8)$ & $224(22.7)$ & \\
\hline Major & 1997 (47.3) & $459(46.5)$ & \\
\hline Extreme & 1129 (26.8) & $282(28.6)$ & \\
\hline Cancer, n (\%) & $1063(25.6)$ & $213(21.7)$ & $0.0106^{*}$ \\
\hline COPD, n (\%) & $628(15.1)$ & $188(19.1)$ & $0.002^{*}$ \\
\hline $\begin{array}{l}\text { Diabetes mellitus, } n \\
(\%)\end{array}$ & 1332 (32.1) & 367 (37.4) & $0.0016^{*}$ \\
\hline CAD, n (\%) & $932(22.5)$ & $213(21.7)$ & 0.606 \\
\hline Stroke, n (\%) & $603(14.5)$ & $172(17.5)$ & $0.019^{*}$ \\
\hline
\end{tabular}

*Statistically significant results, $p<0.05$.

$\mathrm{BMI}$, body mass index; CAD, coronary artery disease; COPD, chronic obstructive pulmonary disease; PcnA, penicillin allergy.

Of those, 1807 patients were missing antibiotic data or did not receive antibiotics during the hospitalisation and were excluded. Of the remaining 5238 patients, 993 (18.9\%) had a reported PcnA label in their EMR and 4245 (81.1\%) did not (figure 1). A higher proportion of patients with reported PcnA were female $(59.3 \%)$ and white $(94.3 \%)$ compared with non-PcnA patients (table 1). Significantly more PcnA compared with non-PcnA patients reported allergic reactions $(4.7 \%$ vs $0 \%, \mathrm{p}<0.0001)$.

Antibiotic use in both groups has been described in figure 2. Cephalosporin use was similar in both groups. In PcnA patients, $29.8 \%$ received cephalosporin antibiotics compared with $31.3 \%$ for non-PcnA patients $(p=0.358)$. Use of other classes of antibiotics (non-PcnA, non-cephalosporin) was more common in PcnA patients $(89.1 \%$ vs $82.7 \%, \mathrm{p}<0.001)$.

Use of multiple antibiotics during hospitalisation was more common in non-PcnA patients, with $29.6 \%$ of patients receiving more than one class of antibiotics compared with $21.9 \%$ of patients in the PcnA group

\begin{tabular}{|l|r|r|r|r|}
\hline Antibiotic class & No PcnA & $\%$ & PcnA & $\%$ \\
\hline Penicillin & 742 & $17.50 \%$ & 31 & $3.10 \%$ \\
\hline Cephalosporin & 1329 & $31.30 \%$ & 296 & $29.80 \%$ \\
\hline Aminioglycoside & 12 & $0.3 \%$ & 6 & $0.6 \%$ \\
\hline Antimycobacterial & 38 & $0.9 \%$ & 3 & $0.3 \%$ \\
\hline Carbapenem & 1 & $0.0 \%$ & 0 & $0.0 \%$ \\
\hline Colistins & 9 & $0.2 \%$ & 4 & $0.4 \%$ \\
\hline Floroquinolone & 1154 & $27.2 \%$ & 336 & $33.8 \%$ \\
\hline Fosfomycin & 16 & $0.4 \%$ & 2 & $0.2 \%$ \\
\hline Glycopeptite (e.g.Vancomycin) & 1513 & $35.6 \%$ & 350 & $35.2 \%$ \\
\hline Licosamide (e.g.Clindamycin) & 373 & $8.8 \%$ & 121 & $12.2 \%$ \\
\hline Macrobid & 15 & $0.4 \%$ & 7 & $0.7 \%$ \\
\hline Macrolide & 611 & $14.4 \%$ & 140 & $14.1 \%$ \\
\hline Nitroimidazoles & 597 & $14.1 \%$ & 159 & $16.0 \%$ \\
\hline Oxazolidinones (e.g.Linezolid) & 37 & $0.9 \%$ & 14 & $1.4 \%$ \\
\hline Sulfonilamides & 11 & $0.3 \%$ & 6 & $0.6 \%$ \\
\hline Tetracyclines & 244 & $5.7 \%$ & 66 & $6.6 \%$ \\
\hline
\end{tabular}

Figure 2 Antibiotic groups used in non-PcnA and PCNA patients. PcnA, penicillin allergy.

$(p<0.0001$, table 2). Antibiotics associated with a higher risk of $C$. difficile infection were more commonly used in the PcnA group (33.8\% received fluoroquinolones compared with $27.2 \%$ in the non-PcnA group, $\mathrm{p}<0.0001)$. A licosamide (clindamycin) was used in $12.2 \%$ of PcnA patients vs $8.8 \%$ of non-PcnA patients $(\mathrm{p}=0.001)$. Glycopeptide (vancomycin) use was similar in both groups (35.2\% for PcnA vs $35.6 \%$ for non-PcnA, $\mathrm{p}=0.814$ ).

Differences were noted in antibiotic use by type and decision to treat with an antibiotic regimen including penicillins for PcnA versus non-PcnA patients $(p<0.05)$. Primary and secondary outcomes between PcnA and non-PcnA patients were not different including 30-day mortality, hospital LOS, antibiotic duration, peripherally inserted central catheter use, hospital-acquired C. difficile infections and total cost of care (table 2). Regression analysis was performed to adjust for patient characteristics to examine outcomes including mortality, hospital LOS, 30-day readmissions, occurrence of $C$. difficile in the hospital, total cost of care and days on antibiotics. Significant differences were not seen among the groups. Therefore, the number needed to treat/harm was not calculated.

After subgroup analysis, out of 993 patients with a reported PcnA, 31 received penicillins and were excluded. The remaining 962 patients were divided into three groups for analysis: those who received cephalosporins only, those who received other classes of antibiotics only, and those who received cephalosporins and other classes of antibiotics simultaneously (figure 1). In a global comparison across the three antibiotic subgroups (table 3), we found differences for age $(p=0.0452)$, severity of illness $(p=0.0089)$ and occurrence of heart failure $(\mathrm{p}=0.041)$.

Further testing was performed to detect differences among the three antibiotics subgroups. Significant differences in age $(p=0.039)$ and incidence of heart failure $(\mathrm{p}=0.047)$ were found between the groups who received other antibiotics and those who received both 
Table 2 Unadjusted outcomes and antibiotics use for hospitalised patients

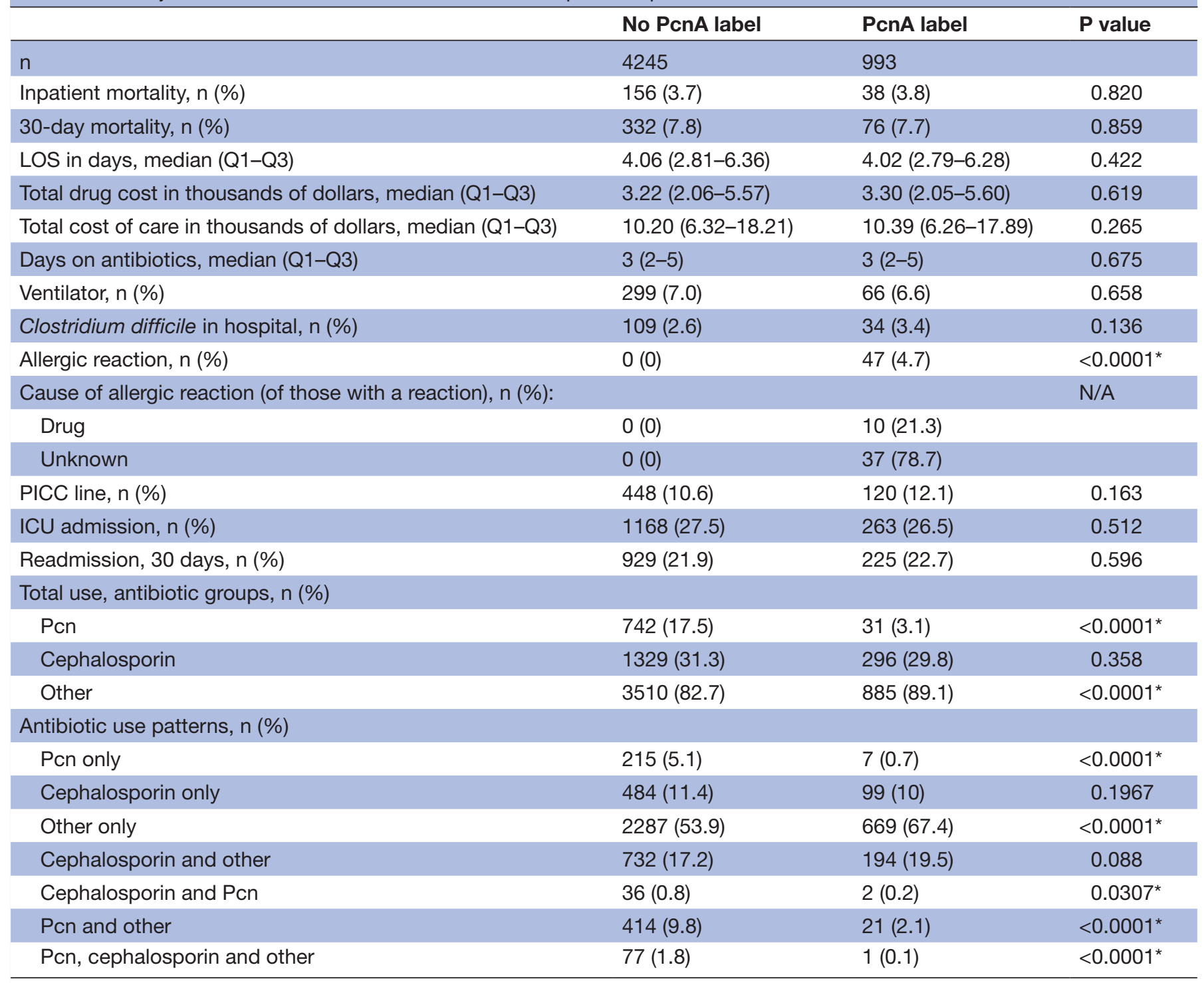

*Statistically significant results $p<0.05$.

ICU, intensive care unit; LOS, length of stay; NA, not available; Pcn, penicillin; PcnA, penicillin allergy; PICC, peripherally inserted central catheter.

cephalosporins and other antibiotics in pairwise comparisons with Bonferroni corrections.

In the subgroup analysis, unadjusted outcomes were assessed by antibiotic type (table 4 ). In a global comparison across the three groups, significant differences were found in median LOS in days $(\mathrm{p}<0.0001)$, median total drug cost in thousands of dollars $(p=0.00393)$, median total cost of care in thousands of dollars $(p=0.000172)$, median days of antibiotics use $(\mathrm{p}<0.0001)$ and occurrence of $C$. difficile in hospital $(\mathrm{p}=0.0138)$. For the readmission logistic regression model, the type of antibiotic administered achieved significance using the Wald test $(p=0.028$, data not shown).

Using an adjusted logistic regression model, no significant differences were found in 30-day mortality across the three antibiotic groups. Using the patient group who received other antibiotics as the reference category, those patients who received both cephalosporins and other antibiotics had higher odds of 30-day readmission ( $\mathrm{OR}=1.54$, 95\% CI 1.04 to 2.27). Patients who received cephalosporins and other antibiotics had a longer hospital LOS (incidence rate ratio, $\mathrm{IRR}=1.25,95 \%$ CI 1.13 to 1.38 ), higher total cost of care (IRR $=1.28,95 \%$ CI 1.12 to 1.46$)$, and more days on antibiotics (IRR=1.59, $95 \%$ CI 1.42 to 1.78 ), (table 5).

\section{DISCUSSION}

We did not find major differences in outcomes between patients with a reported PcnA compared with non-PcnA patients who have documented diagnosis of sepsis, severe sepsis or septic shock. Our study is a retrospective data analysis of electronic health record based queries. We included patients with clinician documented sepsis who 
Table 3 Characteristics of patients with a PCNA label according to type of antibiotic administered

\begin{tabular}{|c|c|c|c|c|}
\hline & Cephalosporin & Other & Cephalosporin and other & $P$ value \\
\hline n & 99 & 669 & 194 & \\
\hline BMI, mean (SD) & $31.8(9.5)$ & $30.7(9.1)$ & $32.5(11.3)$ & 0.0631 \\
\hline Pulse (min), mean (SD) & $67(12)$ & $66(16)$ & $66(13)$ & 0.917 \\
\hline Age, mean (SD) & $65.7(17.7)$ & $64.8(17.7)$ & $68.3(15.5)$ & $0.0452^{*}$ \\
\hline White race, $\mathrm{n}(\%)$ & $95(96.9)$ & $621(93.4)$ & $185(95.9)$ & 0.208 \\
\hline Male, \% (n) & $45(45.5)$ & $264(39.5)$ & $81(41.8)$ & 0.489 \\
\hline Current or former tobacco use, $n(\%)$ & $58(59.2)$ & $386(61.3)$ & $127(69.8)$ & 0.083 \\
\hline Private insurance, n (\%) & 32 (32.3) & $184(27.5)$ & $44(22.7)$ & 0.188 \\
\hline Risk of mortality, n (\%) & & & & 0.052 \\
\hline Minor & $15(15.2)$ & $63(9.5)$ & $18(9.3)$ & \\
\hline Moderate & $26(26.3)$ & $137(20.6)$ & $30(15.5)$ & \\
\hline Major & $27(27.3)$ & $245(36.9)$ & $70(36.3)$ & \\
\hline Extreme & $31(31.3)$ & $219(33)$ & 75 (38.9) & \\
\hline Severity of illness, n (\%) & & & & $0.0089^{\star}$ \\
\hline Minor & $5(5.1)$ & $14(2.1)$ & $3(1.6)$ & \\
\hline Moderate & 32 (32.3) & $151(22.7)$ & $39(20.2)$ & \\
\hline Major & $42(42.4)$ & 307 (46.2) & $91(47.2)$ & \\
\hline Extreme & $20(20.2)$ & $192(28.9)$ & $60(31.1)$ & \\
\hline Cancer, n (\%) & $14(14.1)$ & $154(23.3)$ & $40(20.7)$ & 0.109 \\
\hline Heart failure, n (\%) & 19 (19.2) & $120(18.2)$ & $52(26.4)$ & $0.041^{*}$ \\
\hline COPD, n (\%) & $15(15.2)$ & $127(19.2)$ & $38(19.7)$ & 0.596 \\
\hline CKD, n (\%) & $21(21.2)$ & $137(20.8)$ & $33(17.1)$ & 0.512 \\
\hline Diabetes mellitus, n (\%) & 32 (32.3) & $240(36.4)$ & $83(43)$ & 0.137 \\
\hline Hypertension, n (\%) & $44(44.4)$ & $343(52)$ & $109(56.5)$ & 0.149 \\
\hline CAD, n (\%) & 19 (19.2) & $145(22)$ & $45(23.3)$ & 0.723 \\
\hline Stroke, n (\%) & $16(16.2)$ & $121(18.3)$ & $32(16.6)$ & 0.777 \\
\hline Liver disease, $\mathrm{n}(\%)$ & $6(6.1)$ & $48(7.3)$ & $13(6.7)$ & 0.893 \\
\hline Surgery 4 weeks prior to antibiotic use, $n(\%)$ & $4(4.0)$ & $57(8.5)$ & $12(6.2)$ & 0.207 \\
\hline
\end{tabular}

*Statistically significant results, $\mathrm{p}<0.05$.

$\mathrm{BMI}$, body mass index; CAD, coronary artery disease; CKD, chronic kidney disease; COPD, chronic obstructive pulmonary disease; PcnA, penicillin allergy.

received antibiotics during the hospital visit. Our hypothesis was as far as treating clinician was treating patient for suspected bacterial infection with antibiotics, documented PcnA will affect antibiotic choices and potentially outcomes. Our findings agreed with previously reports for PcnA patients including more use of fluoroquinolones and clindamycin which could have led to more adverse outcomes. However, our non-PcnA group had a larger percentage of patients who received multiple antibiotic classes. Possibly in the PcnA group, fewer classes of antibiotics were administered due to concern for allergic reactions.

Cephalosporins and vancomycin use was similar in both groups. Vancomycin is frequently substituted in patients with reported PcnA in preoperative prophylaxis. ${ }^{24}$ In previous studies, patients with methicillin-susceptible $S$. aureus infection and treated with cefazolin or an anti-staphylococcal PcnA had lower mortality compared with patients treated with vancomycin. ${ }^{25}$ In studies where reported PcnA had adverse outcomes, overuse of vancomycin was implicated as a possible cause. Another explanation for our findings is that the causative pathogen is initially unidentified for many hospitalised sepsis patients, and vancomycin is commonly used to treat a potential MRSA infection especially as initial broad spectrum coverage.

ASPs are expanding in hospitals due to risks associated with inappropriate antibiotic use and Joint Commission standard requirements. ${ }^{26}$ These programmes improve outcomes in patients presenting with severe infections. ${ }^{27}$ At each hospital within our health system, ASPs and PcnA management varied and no ASP had a formal mechanism in place to review PcnA allergies and delabel or challenge PcnA allergies before initiation of antibiotics for sepsis. 
Table 4 Unadjusted outcomes of patients with a PCNA label by antibiotic administered

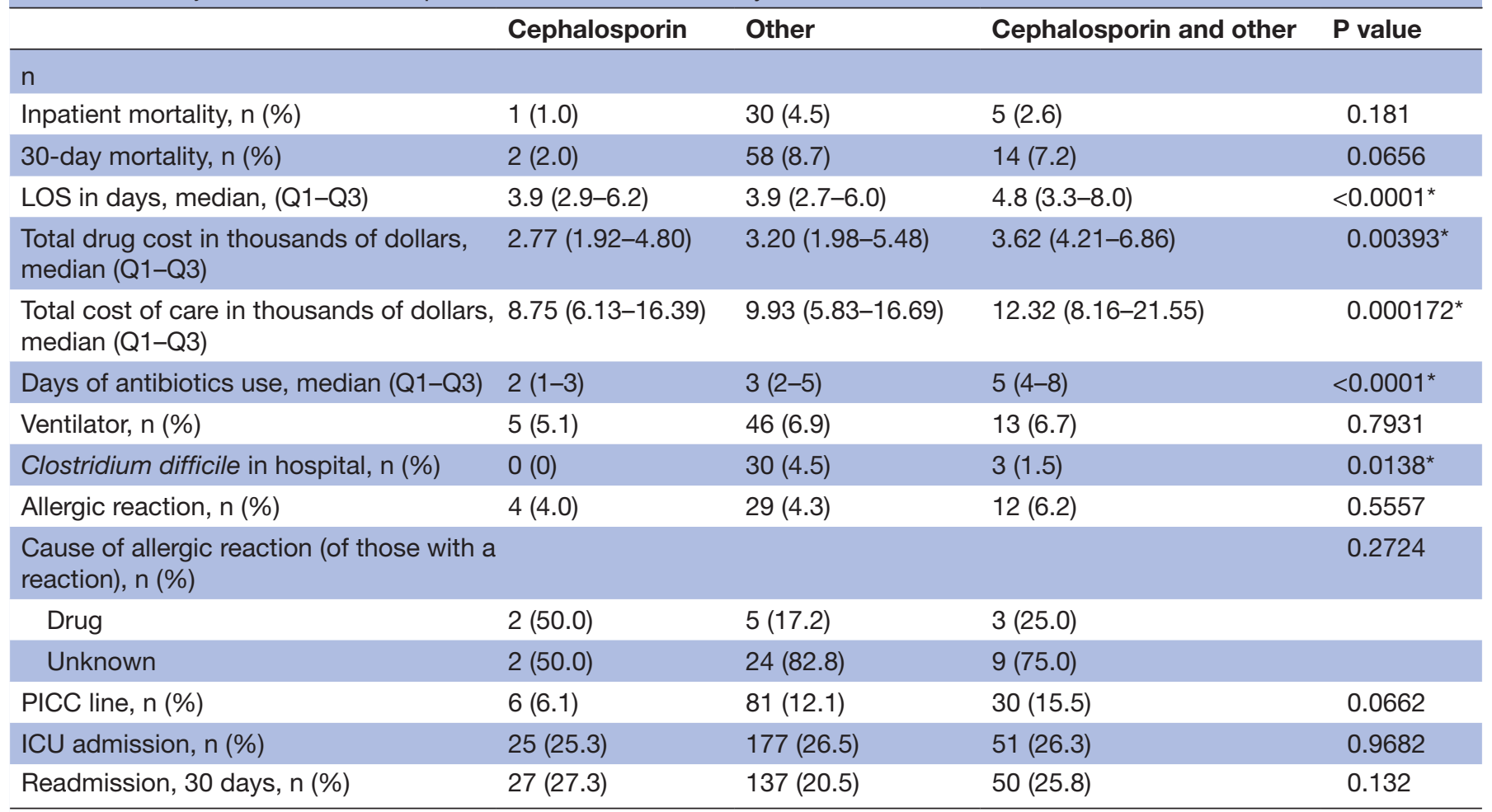

*Statistically significant results, $p<0.05$.

ICU, intensive care unit; LOS, length of stay; PcnA, penicillin allergy; PICC, peripherally inserted central catheter.

PcnA allergies/intolerances that could be safely challenged are often discovered within 48 hours of antibiotic initiation and the reviewing ASP pharmacist or physician, dependent on site, decides whether to change antibiotics for individual patients. Sepsis order sets are reviewed and updated by antimicrobial stewardship staff and infectious disease providers on an annual basis when institutional antibiograms are refreshed. Antibiotics in this order set include source specific sepsis recommendations as well as alternatives in PcnA allergic patients. ASPs likely have a role in our outcomes by influencing providers' choice of antibiotics, especially considering the finding of similar cephalosporin use in both groups.

In the PcnA subgroup analysis, patients who received both cephalosporins and other classes of antibiotics were sicker with a higher severity of illness. This finding reflects common practice where sicker patients get more classes of antibiotics for broader antimicrobial coverage in the early part of hospitalisation. In the patient group receiving only cephalosporins, no patients had hospitalacquired $C$. difficile infection compared with 30 patients $(4.5 \%)$ with this infection who received other antibiotics, but the outcome was not statistically significant. After adjusting for variables including severity of illness, patients who received combined cephalosporin and other antibiotics had an increased hospital LOS, more days on antibiotics and a greater total cost of care. There was no difference in 30-day mortality or 30-day readmissions. Possibly, multiple antibiotic use may play a more significant role than reported PcnA and choice of antibiotics in adverse outcomes among sepsis patients where early use of broad-spectrum antibiotics is common despite reported PcnA. Robust ASPs can also help in optimising antibiotic choices and reducing adverse outcomes.

\section{Limitations}

Our retrospective study relied on EMRs for data accuracy and was at a single healthcare system which may limit generalisability of the findings. We also get large number of patients transferred from hospitals and emergency department outside our health system and some electronic health record is not integrated which lead to exclusion of patients where antibiotic data before transfer to our health system was missing. We did not evaluate time to first antibiotic use. Some studies have suggested adverse outcomes in patients presenting with infection and reported PcnA could be due to a delay in antibiotic administration. Our intention was also to look at realworld situations among all hospitalised patients with diagnosis of sepsis so most of our patients were not very ill and overall mortality was low so this study may not be applicable to sicker patients in intensive care units and could have contributed in detecting mortality outcomes among two groups. We also did not look at reported causes of sepsis due to concern about limited documentation in EMR and reduce in sample size. We also did not categorise patients based on severity of reported PcnA and we did not have data available if patients ever had penicillin 
Table 5 Regression analysis of outcomes of hospitalised patients with a reported PCNA by type of antibiotic administered

\begin{tabular}{|c|c|c|c|}
\hline Regression models & $\begin{array}{l}\text { Unadjusted } \\
\text { OR }\end{array}$ & $95 \% \mathrm{Cl}$ & $P$ value \\
\hline \multicolumn{4}{|l|}{ 30-day readmission } \\
\hline Cephalosporin only & 1.49 & 0.90 to 2.38 & 0.106 \\
\hline $\begin{array}{l}\text { Cephalosporin and } \\
\text { other }\end{array}$ & 1.38 & 0.94 to 2.00 & 0.091 \\
\hline \multicolumn{4}{|l|}{ 30-day mortality } \\
\hline Cephalosporin only & 0.27 & 0.04 to 0.80 & $0.045^{\star}$ \\
\hline $\begin{array}{l}\text { Cephalosporin and } \\
\text { other }\end{array}$ & 0.78 & 0.39 to 1.40 & 0.429 \\
\hline
\end{tabular}

\begin{tabular}{|c|c|c|c|}
\hline & Adjusted OR & $95 \% \mathrm{Cl}$ & $P$ value \\
\hline \multicolumn{4}{|l|}{ 30-day readmission } \\
\hline Cephalosporin only & 1.65 & 0.99 to 2.68 & 0.049 \\
\hline Cephalosporin and other & 1.54 & 1.04 to 2.27 & $0.03^{*}$ \\
\hline \multicolumn{4}{|l|}{ 30-day mortality } \\
\hline Cephalosporin only & 0.35 & 0.05 to 1.08 & 0.113 \\
\hline \multirow[t]{2}{*}{ Cephalosporin and other } & 0.70 & 0.34 to 1.29 & 0.267 \\
\hline & $\begin{array}{l}\text { Incidence } \\
\text { rate ratio }\end{array}$ & $95 \% \mathrm{Cl}$ & $P$ value \\
\hline \multicolumn{4}{|l|}{ Hospital LOS } \\
\hline Cephalosporin only & 1.11 & 0.97 to 1.27 & 0.135 \\
\hline Cephalosporin and other & 1.25 & 1.13 to 1.38 & $<0.001^{*}$ \\
\hline \multicolumn{4}{|c|}{ Total cost of care } \\
\hline Cephalosporin only & 1.13 & 0.95 to 1.35 & 0.187 \\
\hline Cephalosporin and other & 1.28 & 1.12 to 1.46 & $<0.001^{*}$ \\
\hline \multicolumn{4}{|l|}{ Days on antibiotics } \\
\hline Cephalosporin only & 0.87 & 0.74 to 1.03 & 0.115 \\
\hline Cephalosporin and other & 1.59 & 1.42 to 1.78 & $<0.001^{*}$ \\
\hline
\end{tabular}

Other antibiotics group is the reference category.

*Statistically significant results, $\mathrm{p}<0.05$.

LOS, length of stay; PcnA, penicillin allergy.

skin testing. We only looked at antibiotics administered during the hospitalisation and did not review discharge prescriptions which also could be a limiting factor.

\section{CONCLUSION}

Despite limitation our study suggests that hospitalised patients with reported PcnA who had documented sepsis, severe sepsis or septic shock and received antibiotics did not have worse outcomes compared with Non-PcnA. Use of multiple classes of antibiotics was common in both groups. Antibiotics with higher risk of $C$. difficile were used more commonly with reported PcnA. More research is needed to evaluate the impact of reported PcnA in patients hospitalised with infections.

Contributors DB provided conceptualisation, methodology, design, clinical interpretation and writing, review and editing of the submitted manuscript. LP contributed to methodology, design, clinical interpretation and writing, review, editing of the submitted manuscript and revision of manuscript. CSS performed data collection, analysis, and writing, review, and editing of the submitted manuscript. JK contributed to methodology, design, clinical interpretation, and writing, review, and editing of the submitted manuscript. CS participated in study development, and writing, review, and editing of the submitted manuscript. DR participated in study development, clinical interpretation, and writing, review, and editing of the submitted manuscript. RK contributed in revision of manuscript. MW participated in study development, clinical interpretation and writing, review, and editing of the submitted manuscript. BD contributed to study development, and writing, review and editing of the submitted manuscript. CASH supervised data collection and contributed to methodology, project design, analysis and interpretation, and editing of the submitted manuscript. The authors would like to acknowledge the contributions of JK to data extraction from electronic medical records. DB as guarantor accepts full responsibility for the work and/or the conduct of the study, had access to the data, and controlled the decision to publish.

Funding The authors have not declared a specific grant for this research from any funding agency in the public, commercial or not-for-profit sectors.

Competing interests None declared.

Patient and public involvement Patients and/or the public were not involved in the design, or conduct, or reporting, or dissemination plans of this research.

Patient consent for publication Not applicable.

Ethics approval Allina Health's Institutional Review Board (IRB) approved this study with a waiver of informed consent, ID \# 1380327.

Provenance and peer review Not commissioned; externally peer reviewed.

Data availability statement Data are available on reasonable request. Deidentified participant data were used for this research study.

Open access This is an open access article distributed in accordance with the Creative Commons Attribution Non Commercial (CC BY-NC 4.0) license, which permits others to distribute, remix, adapt, build upon this work non-commercially, and license their derivative works on different terms, provided the original work is properly cited, appropriate credit is given, any changes made indicated, and the use is non-commercial. See: http://creativecommons.org/licenses/by-nc/4.0/.

\section{ORCID iDs}

Love Patel http://orcid.org/0000-0002-6271-6376

Catherine A St. Hill http://orcid.org/0000-0002-6113-5705

\section{REFERENCES}

1 Cecconi M, Evans L, Levy M, et al. Sepsis and septic shock. The Lancet 2018;392:75-87.

2 Singer M, Deutschman CS, Seymour CW, et al. The third International consensus definitions for sepsis and septic shock (Sepsis-3). JAMA 2016;315:801-10.

3 Angus DC, Linde-Zwirble WT, Lidicker J, et al. Epidemiology of severe sepsis in the United States: analysis of incidence, outcome, and associated costs of care. Crit Care Med 2001;29:1303-10.

4 Arefian $\mathrm{H}$, Heublein S, Scherag A, et al. Hospital-related cost of sepsis: a systematic review. J Infect 2017;74:107-17.

5 Torio CM, Moore BJ. National Inpatient Hospital Costs: The Most Expensive Conditions by Payer, 2013: Statistical Brief \#204. In: Healthcare Cost and Utilization Project (HCUP) Statistical Briefs. US Agency for Healthcare Research and Quality, 2006. http://www.ncbi. nlm.nih.gov/books/NBK368492/

6 Iwashyna TJ, Ely EW, Smith DM, et al. Long-Term cognitive impairment and functional disability among survivors of severe sepsis. JAMA 2010;304:1787-94.

7 Rhodes A, Evans LE, Alhazzani W, et al. Surviving sepsis campaign: international guidelines for management of sepsis and septic shock: 2016. Crit Care Med 2017;45:486-552.

8 Ferrer R, Martin-Loeches I, Phillips G, et al. Empiric antibiotic treatment reduces mortality in severe sepsis and septic shock from the first hour: results from a guideline-based performance improvement program. Crit Care Med 2014;42:1749-55.

9 Seymour CW, Gesten F, Prescott HC, et al. Time to treatment and mortality during mandated emergency care for sepsis. N Engl $J$ Med 2017;376:2235-44.

10 Jacobs ZG, Prasad PA, Fang MC, et al. The association between limited English proficiency and sepsis mortality. J Hosp Med 2019;14:E1-7.

11 Lee CE, Zembower TR, Fotis MA, et al. The incidence of antimicrobial allergies in hospitalized patients: implications regarding 
prescribing patterns and emerging bacterial resistance. Arch Intern Med 2000;160:2819-22.

12 Macy E. Penicillin allergy: optimizing diagnostic protocols, public health implications, and future research needs. Curr Opin Allergy Clin Immunol 2015;15:308-13.

13 Gadde J, Spence M, Wheeler B, et al. Clinical experience with penicillin skin testing in a large inner-city STD clinic. JAMA 1993;270:2456-63.

14 Shenoy ES, Macy E, Rowe T. Evaluation and management of PCNA: a review. JAMA 2019;321:188-99.

15 Crotty DJ, Chen XJC, Scipione MR, et al. Allergic reactions in hospitalized patients with a self-reported penicillin allergy who receive a cephalosporin or meropenem. J Pharm Pract 2017;30:42-8.

16 Har D, Solensky R. Penicillin and beta-lactam hypersensitivity. Immunol Allergy Clin North Am 2017;37:643-62.

17 Campagna JD, Bond MC, Schabelman E, et al. The use of cephalosporins in penicillin-allergic patients: a literature review. $J$ Emerg Med 2012;42:612-20.

18 Blumenthal KG, Peter JG, Trubiano JA, et al. Antibiotic allergy. Lancet 2019;393:183-98.

19 Macy E, Contreras R. Health care use and serious infection prevalence associated with penicillin "allergy" in hospitalized patients: A cohort study. Journal of Allergy and Clinical Immunology 2014;133:790-6.
20 Conway EL, Lin K, Sellick JA, et al. Impact of penicillin allergy on time to first dose of antimicrobial therapy and clinical outcomes. Clin Ther 2017;39:2276-83.

21 Blumenthal KG, Ryan EE, Li Y, et al. The impact of a reported penicillin allergy on surgical site infection risk. Clin Infect Dis 2018;66:329-36.

22 Pollack LA, Srinivasan A. Core elements of hospital antibiotic stewardship programs from the centers for disease control and prevention. Clin Infect Dis 2014;59 Suppl 3:S97-100.

23 del Real GA, Rose ME, Ramirez-Atamoros MT, et al. Penicillin skin testing in patients with a history of $\beta$-lactam allergy. Annals of Allergy, Asthma \& Immunology 2007;98:355-9.

24 Joint Task Force on Practice Parameters, American Academy of Allergy, Asthma and Immunology, American College of Allergy, Asthma and Immunology, et al. Drug allergy: an updated practice parameter. Ann Allergy Asthma Immunol 2010;105:259-73.

25 Verani JR, McGee L, Schrag SJ, et al. Prevention of perinatal group B streptococcal disease--revised guidelines from CDC, 2010. MMWR Recomm Rep 2010;59:1-3.

26 Joint Commission on Hospital Accreditation. Approved: new antimicrobial stewardship standard. Jt Comm Perspect 2016;36:3-4.

27 Sherbuk JE, McManus D, Topal JE, et al. Improved mortality in Staphylococcus aureus bacteremia with the involvement of antimicrobial stewardship team and infectious disease consultation. Infect Control Hosp Epidemiol 2019;40:932-5. 